Helgoländer wiss. Meeresunters. 20, 249-263 (1970)

\title{
Die Zucht von niederen marinen Wirbellosen und ihre Anwendung in der experimentellen Zoologie
}

\author{
C. HAuenschild \\ Zoologisches Institut der Technischen Universität Braunscbweig; \\ Braunschweig, Deutschland (BRD)
}

\begin{abstract}
Cultivation of lower marine invertebrates and its application in experimental zoology. This paper summarizes the present status of our knowledge concerning the cultivation of lower marine invertebrates. On the basis of the author's own work and experience, the term "culture" is defined and the advantages of continuous laboratory cultivation for experimental investigations are considered. The prerequisites for successful cultivation and containers, suitable for cultivating small marine invertebrates, are discussed. Special attention is paid to the selection of species for culture work and to the major factors known to be important in regard to culture techniques (seawater, Oz-supply, water movement, food, temperature, light). Further points considered are: provisions for maintenance of a biological steady-state system in cultures; mono- and polyxenic cultures. Some examples of scientific results obtained by cultivation of marine invertebrates are presented.
\end{abstract}

\section{EINLEITUNG}

Der Begriff „Zucht“ oder Kultur wird häufig in einem etwas zu weit gefaßten Sinn gebraucht. Man sollte ihn in der wissenschaftlichen Biologie möglichst auf die Fälle beschränken, in denen folgende Bedingungen verwirklicht sind:

(1) Der gezüchtete Organismus pflanzt sich unter künstlichen Bedingungen geschlechtlich oder ungeschlechtlich über beliebig viele Generationen fort.

(2) Diese Bedingungen sind soweit überschaubar und definiert, daß sie sich jederzeit reproduzieren und über Jahre aufrechterhalten lassen.

Damit ist die Zucht, bei der nicht so sehr die Erhaltung des Einzelindividuums als die eines fortlaufend sich erneuernden Bestandes im Vordergrund steht, klar gegen die sogenannte Hälterung abgegrenzt. Von Hälterung spricht man, wenn Organismen für eine begrenzte Zeit unter künstlichen Bedingungen am Leben erhalten werden und wenn sie sich dabei gar nicht oder nur so unzureichend fortpflanzen, daß der Bestand auf die Dauer nicht ohne Zufuhr neuer Individuen von außen erhalten werden kann. Dieser Beitrag beschränkt sich auf die Zucht vielzelliger, wirbelloser Meerestiere, wobei die sogenannten niederen Tierstämme im Vordergrund stehen sollen. Außer Betracht bleibt die Tierzucht im großen Stil, wie sie z. B. bei einigen Mollusken und Crustaceen zum $Z$ weck der wirtschaftlichen Verwertung betrieben wird; es soll demgemäß 
nur von Laborzuchten, soweit sie der zoologischen Grundlagenforschung dienen, die Rede sein (vgl. Hauenschild 1962, 1968).

\section{VORZÜGE UND VORAUSSETZUNGEN KONTINUIERLICHER ZUCHTEN}

Zahlreiche an lebenden Tieren vorzunehmende Untersuchungen, etwa auf verschiedenen Gebieten der Physiologie oder der Ethologie, lassen sich ohne weiteres an einigen Individuen durchführen, die nur vorübergehend im Labor gehalten werden. Dagegen bedeutet es für viele andere Fragestellungen, z. B. biorhythmischer, fortpflanzungs- oder entwicklungsbiologischer Art, eine wesentliche methodische Bereicherung, wenn sie auf der Basis von Laborzuchten bearbeitet werden können. Dabei ist vor allem an die folgenden Vorteile zu denken: Der gesamte Lebenszyklus einer Art läßt sich in den Kulturen kontinuierlich verfolgen; die Vorgeschichte jedes Versuchstiers ist bekannt; die Materialbeschaffung ist von der Jahreszeit unabhängig und in der Regel stehen alle Entwicklungsstadien gleichzeitig zur Verfügung. Schließlich gibt es auch Arbeitsmethoden, die ohne eine kontinuierliche Zucht des Versuchsobjekts überhaupt nicht anwendbar sind, wie z. B. das Kreuzungsexperiment. Eine Möglichkeit, von der bisher noch kaum Gebrauch gemacht wurde, ist die Anwendung von Kulturmethoden für ökologische Modellversuche, mit denen die Zusammenhänge in einfachen Okosystemen einer experimentellen Analyse zugänglich sein könnten. Nicht zuletzt lassen sich Zuchten mariner Invertebraten als Lieferanten lebenden Untersuchungs- und Demonstrationsmaterials in den Dienst des Hochschulunterrichts stellen; dadurch wird es möglich, auch diejenigen oft besonders wichtigen Tiergruppen, die im Binnenland nicht vorkommen, in das Kursprogramm einzubeziehen.

Aus praktischen Gründen sind an eine Laborzucht verschiedene Ansprüche zu stellen, welche den Kreis der für die Kultur geeigneten Tierarten von vornherein einengen. Der räumliche und apparative Aufwand muß sich soweit in Grenzen halten, $\mathrm{daß}$ er in einer vernünftigen Relation zum wissenschaftlichen Ertrag steht. Die gezüchtete Tierart soll eine möglichst schnelle Generationsfolge aufweisen, wie sie besonders für genetische und entwicklungsphysiologische Arbeiten erwünscht ist. Außerdem muß die Zucht einen genügend großen Vermehrungsüberschuß produzieren, damit laufend Individuen für Versuchszwecke entnommen werden können. Die Tiere sollen sich in den Kulturgefäßen beobachten lassen, erforderlichenfalls auch mit Lupe oder Stereomikroskop. Schließlich müssen alle für den Zuchterfolg wesentlichen Komponenten überschaubar bleiben, damit der kontinuierliche Bestand der Kultur und die Reproduzierbarkeit der Methode gewährleistet sind. Die meisten dieser Bedingungen begrenzen gleichermaßen die Größe der zu züchtenden Arten wie auch das Volumen der Zuchtbehälter.

\section{Zuchtgef äße}

In größeren Aquarien ist im allgemeinen weder eine ausreichende Beobachtungsmöglichkeit für kleine Tiere noch die erforderliche Úberschaubarkeit der Bedingungen gegeben. Man ist daher gezwungen, sich möglichst kleiner Zuchtgefäße zu bedienen 
(Abb. 1) und den Nachteil des knapp bemessenen Wasservolumens durch verschiedene konservierende Maßnahmen auszugleichen. Für Tiere im mm-Bereich, die ohne Durchlïftung auskommen, eignen sich Boveri- und Petrischalen, für etwas größere Arten Kristallisierschalen mit gewölbtem oder flachem Boden. Entweder wird jede Schale für sich mit einem Deckel versehen, oder es können auch mehrere offene Schalen gemeinsam

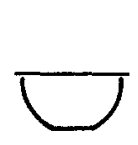

$a$

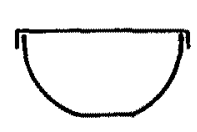

$b$

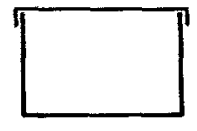

C

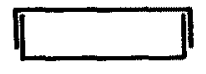

$d$

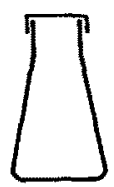

e

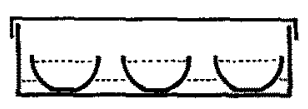

f

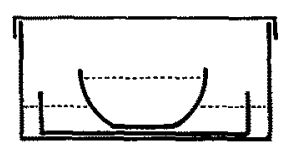

G
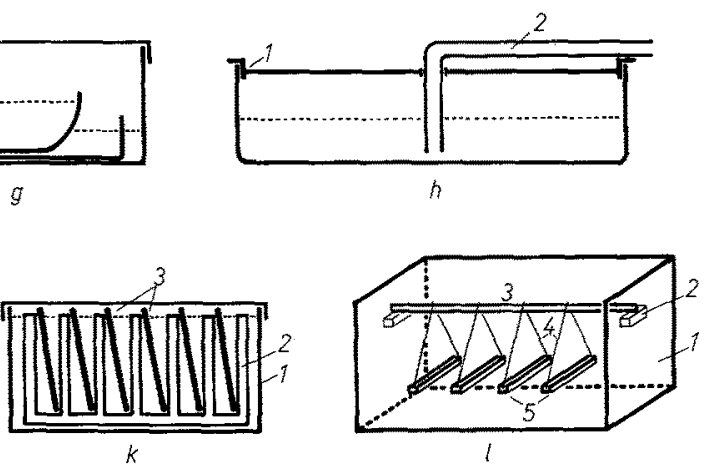

Abb. 1: Zuchtgefäße. $a=$ Boverischale mit Deckel aus Fensterglas; $b=$ halbkugelige Kristallisierschale mit einer halben Petrischale als Deckel; $c=$ zylindrische Kristallisierschale; $d=$ Petrischale; $e=$ Weithals-Erlenmeyerkolben mit kleiner, halber Petrischale als Deckel; $f=$ kleine, offene Kristallisierschälchen in einer großen, als feuchte Kammer fungierenden Petrischale; $g=$ Satz von 3 ineinandergestellten Schalen (die innerste enthält Tiere, die häufig aus dem Wasser herauskriechen); $b=$ durchlüftete Polystyrolbox: $1=$ Deckel mit Spritzwasserfalz und zentraler Bohrung, $2=$ Durchlüftungsrohr; $i=$ Vorrichtung zur kontinuierlichen Fütterung von Hydroiden: $1=$ Polystyrolbox, $2=$ Petrischale mit Artemia-Larven, $3=$ Heberrohr, $4=$ Petrischale mit Hydroidpolypen; $k=$ Kultur festsitzender Tiere auf Objektträgern: $1=$ zylindrische Kristallisierschale, $2=$ Glasstab-Gestell, $3=$ mit Tieren besetzte Objektträger; $l=$ Kultur festsitzender Tiere auf Glasstäbchen: $1=$ Polystyrolbox, $2=$ Halterung, $3=$ Glasstab, $4=$ Perlonfäden, $5=$ mit Tieren besetzte Glasstäbchen. (Nach HaUENSChILd 1968)

in ein größeres, geschlossenes Gefäß, welches als feuchte Kammer fungiert, hineingestellt werden. Tiere, die häufig aus dem Wasser herauskriechen, werden am zweckmäßigsten in der innersten von 3 ineinandergestellten Schalen untergebracht und in regelmäßigen Abständen aus den äußeren, ebenfalls mit Seewasser gefüllten Gefäßen wieder in das mittlere zurückbefördert. In den genannten Schalen läßt sich eine Kultur prinzipiell in ihrer ganzen räumlichen Ausdehnung unter einem Stereomikroskop kontrollieren. Kolben oder Flaschen wird man in der Regel nur zur Zucht freischwimmender, autotropher Flagellaten, wie sie als Futter für Tierkulturen dienen, verwenden. Als Behälter für Zuchten, die durchlüftet werden müssen, sind Kühlschrankgefäße aus 
Polystyrol sehr praktisch; ihre Deckel lassen sich zwecks Einführung eines Durchlüftungsröhrchens leicht durchbohren und besitzen außerdem am Rand einen Falz, der den Austritt von Spritzwasser und das Ausblühen von Salz aus der Schale weitgehend verhindert. Unter dem Binokularmikroskop sind diese Schalen $z$ war etwas unhandlich, bei Zuhilfenahme eines Präparierstativs mit Schwenkarm ist aber eine Untersuchung der darin befindlichen Tiere ohne weiteres möglich. Für sessile Tiere wie Hydrozoen, Kamptozoen oder Bryozoen, kann es zur Vermeidung einer schädlichen Verschmutzung angezeigt sein, die Kolonien auf Objektträgern anzusiedeln, die aufrecht in einem Glas- oder Plastikgestell stehen, oder auf Glasstäbchen, die an Perlonfäden frei in einer Schale aufgehängt sind. Als Material für die Zuchtgefäße kommen Glas und verschiedene Kunststoffe in Betracht; die letzteren müssen allerdings von Fall zu Fall hinsichtlich ihrer Unschädlichkeit für eine bestimmte Tierart ausprobiert werden.

\section{Auswahl der Zuchtobjekte}

Im Hinblick auf räumliche Begrenzung und kurze Generationsdauer wird man bei der Auswahl der Zuchtobjekte in den meisten Tiergruppen den jeweils kleineren Species den Vorzug geben. Unabhängig davon sind natürlich immer diejenigen Arten am leichtesten züchtbar, die am wenigsten spezifische Ansprüche stellen und die eine größere Toleranz gegenüber Schwankungen der Lebensbedingungen aufweisen. Solche Formen finden sich vorzugsweise im Litoral; so zeigen etwa die Bewohner von Gezeitentümpeln im Felswatt vielfach eine erhebliche Resistenz, die sie in besonderem Maße für die Zucht prädestiniert. Auch bei solchen Tierarten kann es Schwierigkeiten geben, falls die Individuen untereinander unverträglich sind und sich gegenseitig verstümmeln, sobald sie in großer Zahl auf engem Raum zusammenleben müssen. Soweit es sich um Kleinlebewesen im $\mu \mathrm{m}$ - oder mm-Bereich handelt, sind 2 verschiedene Wege für den Aufbau neuer Kulturen gangbar.

Der eine besteht darin, aus dem Meer eingebrachte Materialproben im Labor einige Zeit unter verschiedenen Standardbedingungen zu halten und auf diese Weise diejenigen Tierarten zu selektionieren, die bei den betreffenden Bedingungen gedeihen. Man kann z. B. eine Portion der Probe regelmäßig mit Artemia-Larven versehen, eine zweite mit einer Flagellatensuspension und eine dritte mit fein zerriebenem Aquarienfisch-Futter; in jedem Fall werden sich im Laufe von Wochen oder Monaten andere Tiere entwickeln und anreichern, und zwar jeweils solche, die sich von dem zugesetzten Futter direkt oder indirekt zu ernähren vermögen. Entsprechend ist auch eine Auswahl in bezug auf andere Faktoren möglich. Man braucht die selektionierten Arten dann nur noch in einer ausreichenden Individuenzahl aus der Materialprobe mit Hilfe einer Pipette zu isolieren und mittels einer mehrmaligen Passage durch Schälchen mit sterilem Seewasser von Verunreinigungen zu befreien, um sie dann mit der bereits ausgearbeiteten Standardmethode weiterkultivieren zu können. Dieses Verfahren fördert allerdings nur ein relativ begrenztes Spektrum besonders vitaler Formen zutage, da sich die anspruchsvolleren Arten in solchen der selbsttätigen Auslese unterworfenen Materialproben nur selten durchsetzen können.

Um empfindlichere oder größere Arten in Kultur zu nehmen, muß man den zwei- 
ten Weg gehen, der allerdings gewöhnlich sehr viel mühsamer ist. Man isoliert hierbei die erforderliche Anzahl von Exemplaren der gewünschten Tierart sofort aus dem daraufhin durchgesuchten Freilandmaterial, reinigt die Tiere und probiert dann in mehreren parallel laufenden Versuchsreihen systematisch aus, welches ihre essentiellen Lebensansprüche sind. Gewöhnlich ist es günstig, dabei nicht von beliebigen Individuen, sondern von geschlechtsreifen Tieren, eiertragenden Weibchen oder Gelegen auszugehen, damit man von vornherein die Zuchtbedingungen nicht nur für die Erwachsenen, sondern auch für die frühen Entwicklungsstadien ermitteln kann.

\section{DIE ROLLE ABIOTISCHER UND BIO'TISCHER FAKTOREN}

Die Parameter, die für das Funktionieren einer Zucht entscheidend sind, betreffen vor allem das Kulturmedium, die Wasserbewegung und $\mathrm{O}_{2}$-Versorgung, das Futter, die Temperatur und das Licht.

\section{Kulturmedium}

Als Kulturflüssigkeit kommt natürliches oder synthetisches Seewasser oder eine Mischung von beiden in Betracht. Für empfindlichere Tiere oder bestimmte Entwicklungsstadien ist natürliches Seewasser dem künstlichen immer noch überlegen, was vermutlich auf seinen Gehalt an organischen, im einzelnen noch nicht näher analysierten Substanzen zurückzuführen ist. Ungünstig wirkt sich nach meinen Erfahrungen eine länger andauernde Aufbewahrung natürlichen Seewassers in Polyäthylen-Behältern aus. Dichteunterschiede, wie sie z. B. zwischen Nordsee- und Mittelmeerwasser bestehen, ertragen die meisten Tiere ohne weiteres; ein zu starkes Ansteigen der Salzkonzentration, wie es in unbedeckten Gefäßen ziemlich rasch eintritt, muß desungeachtet natürlich vermieden werden. Hinsichtlich des $\mathrm{pH}$ kann ein Wert zwischen 7,6 und 8,0 als Norm gelten. In jedem Fall ist die Kulturflüssigkeit, vor allem bei Verwendung natürlichen Wassers, vor Gebrauch durch Erhitzen zu sterilisieren. Sofern nicht aus besonderen Gründen Bakterienfreiheit angestrebt wird, genügt ein- oder zweimaliges Erwärmen auf $80^{\circ} \mathrm{C}$, um alle diejenigen Organismen, die eine Kultur durch Massenvermehrung zerstören können, auszuschalten; es sind dies u. a. verschiedene Amoeben, Ciliaten, Diatomeen, Blaualgen und Pilze. Wenn man die im Seewasser stets suspendierten Feststoffe von den Kulturen fernhalten will, um die Bildung eines oft unerwünschten Bodenbelages zu verhindern, ist es erforderlich, das Seewasser vor der Sterilisierung abklären zu lassen oder zu filtrieren.

\section{Belüftung und Wasserbewegung}

Zahlreichen Tieren genügt ein Gasaustausch, wie er sich durch Diffusion in einer undurchlïfteten, zugedeckten Kulturschale vollzieht, sofern eine Übervölkerung der Kultur verhindert wird. In anderen Fällen muß der Gasaustausch durch besondere Maßnahmen intensiviert werden. Manchmal reicht es schon aus, wenn man das Wasser 
in den Zuchtschalen nicht höher als etwa $5 \mathrm{~mm}$ stehen läßt. Am wirksamsten ist natürlich eine ständige Umschichtung des Kulturmediums entweder durch Einleiten von Luftblasen oder durch eine langsam laufende Schüttelapparatur (Abb. 2). Als solche hat sich ein Gestell bewährt, in dem mehrere Tabletts übereinander angeordnet sind und das auf 4 Kugellagern in 2 Gleitschienen läuft: es wird von einem Getriebemotor über eine exzentrisch gelagerte Schubstange ungefähr 25mal pro Min. mit einer Ampli-
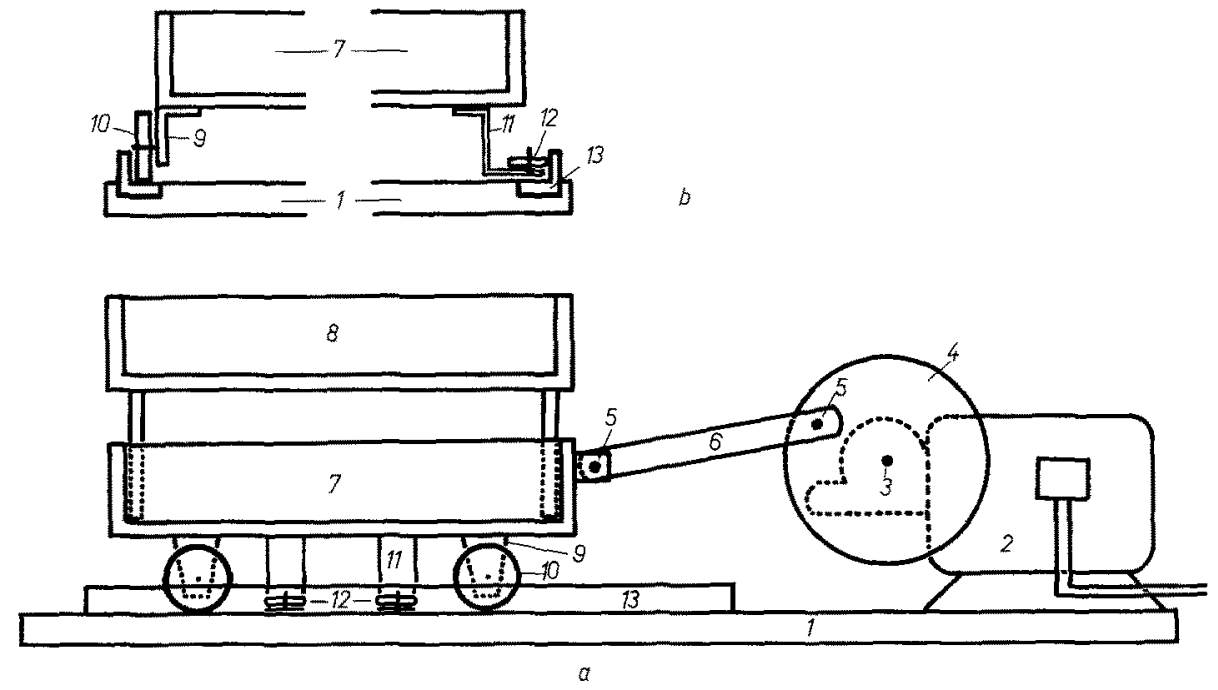

Abb. 2: Langsam laufende Schüttelapparatur. $a=$ Seitenansicht, $b=$ Querschnitt (links in Höhe des Kugellagers, rechts in Höhe des Führungsrädchens): $1=$ Grundplatte, $2:=$ Elektromotor (1360 U/min, $50 \mathrm{~W}), 3=$ Antriebsachse des Getriebevorsatzes $(55: 1), 4=$ Scheibenrad ( $\varnothing 10 \mathrm{~cm}), 5=$ Lagerzapfen, $6=$ Schubstange, $7=$ Wagen, $8=$ abnehmbarer Aufsatz (mehrere Stockwerke möglich), $9=$ Halterung für Kugellagerachse, $10=$ Kugellager, $11=$ Halterung für die Achse des Führungsrädchens, $12=$ Führungsrädchen, $13=$ Stahlschiene.

(Nach Hauenschrmd 1968)

tude von etwa $10 \mathrm{~cm}$ hin und her bewegt. Die Stärke der dadurch verursachten Wasserbewegung läßt sich durch Größe und Form der Zuchtgefäße sowie durch den Wasserstand in denselben beeinflussen. Abgesehen von der $\mathrm{O}_{2-}$ Versorgung ist eine Wasserbewegung bei vielen sessilen Tieren auch insofern wichtig, als sie einer übermäßigen Verschmutzung der Tiere durch sedimentierende Abfallstoffe entgegenzuwirken vermag.

\section{Futterorganismen}

Am leichtesten sind Tiere zu züchten, die sich direkt mit einem autotrophen, in Reinkultur rasch zu vermehrenden Organismus ernähren lassen. Für diesen Zweck stehen heute zahlreiche Arten von Flagellaten, Diatomeen und Grünalgen, die bereits als Reinkulturen existieren oder zumindest schon vorher erfolgreich gezüchtet worden sind, zur Auswahl. Je nach der Ernährungsweise des zu kultivierenden Tieres können 
z. B. innerhalb der Flagellaten rasenbildende Formen wie Platymonas oder überwiegend freischwimmende Arten wie Dunaliella oder Cryptomonas verwendet werden.

Unter den heterotrophen Einzellern ist besonders der phagotrophe, auf einfache Weise mit Dunaliella zu ernährende Dinoflagellat Oxyrrbis günstig, da er von vielen Tieren gern gefressen wird und offenbar leicht verdaulich ist. Als Ergänzung zu der rein pflanzlichen Kost hat sich bei manchen Tieren (z. B. Platynereis) ein Zusatz von Trockenfutter, wie es für Aquarienfische gebräuchlich ist, gut bewährt. Manche der sogenannten Detritusfresser füttert man statt mit lebenden besser mit hitzegetöteten Flagellaten, die in der Schale einen Bodenbelag bilden und die Bakterienentwicklung anregen; z. B. gedeiht Dinopbilus oder Ctenodrilus mit einer Dunaliella, die vorher auf $50^{\circ} \mathrm{C}$ erhitzt wurde.

Für carnivore Tiere kommen als Standardnahrung in erster Linie die Nauplien von Artemia salina in Betracht. Es empfiehlt sich dabei, die frischgeschliuptten, sehr fettreichen Larven vor der Verfütterung einige Tage hungern zu lassen, um den Fettgehalt herabzusetzen; die Nauplien werden dann, vor allem bei andauernder Verwendung als alleiniges Futter, von den meisten Tieren viel besser vertragen. Artemia läßt sich nach Abtötung bei $50^{\circ} \mathrm{C}$ auch gut als Nahrung für Aasfresser (z. B. Opbryotrocha) verwenden; dabei ist natürlich besonders auf knappe Dosierung zu achten. In gleicher Weise sind Tubifex, Mückenlarven, Daphnien oder Mysis, die heute auch tiefgefroren oder gefriergetrocknet erhältlich sind, anwendbar. Als Lebendfutter kommen ferner Enchytraeen in Betracht, da sie in Seewasser kurze Zeit lebendig bleiben, sowie Miesmuscheln, deren Fleisch sich vor allem zur Ernährung größerer Tiere eignet. Für viele kleinere Tiere fehlt leider immer noch ein geeigneter, leicht züchtbarer mariner Futterorganismus, der in der Größe zwischen Oxyrrhis und Artemia-Nauplius steht.

Bei manchen Tieren ist es nicht zu umgehen, daß man für die Zucht eine mehrgliedrige Nahrungskette aufbaut. Beispielsweise frißt der Polychaet Autolyins nur Hydroiden, die ihrerseits mit Artemia als Futter laufend herangezogen werden müssen; falls man die Artemien mit Flagellaten als Nahrung selbst züchtet (was angesichts der käuflichen Artemia-Eier als Routinemethode allerdings kaum lohnend erscheint), liegt sogar ein dreigliedriges System vor. Der günstigste Zeitabstand zwischen zwei Fütterungen ist bei jeder Tierart ein anderer und kann nur empirisch festgestellt werden; eine Nahrungszufuhr kann alle 1-2 Tage, wöchentlich einmal oder auch nur alle 2-3 Wochen erforderlich sein. Allgemein, ganz besonders aber bei Fleischfressern, empfiehlt es sich, lieber knappe Futterdosen in kürzeren Intervallen als reichliche Gaben in längeren Abständen zu verabreichen.

\section{Temperatur}

Toleranzgrenzen und Optimum hinsichtlich der Zuchttemperatur sind in erster Linie durch die Herkunft der Tiere bestimmt. Am besten wäre es, für die Meerestiere der verschiedenen Klimazonen bzw. Tiefenstufen verschiedene Zuchträume zu haben, in denen die Temperatur auf unterschiedlichen Werten durch Klimageräte konstant gehalten wird; man würde sie dann für Litoraltiere aus tropischen Gebieten auf $26^{\circ}$ bis $28^{\circ} \mathrm{C}$ einstellen, für solche aus dem Mittelmeer auf $20^{\circ}$ bis $22^{\circ} \mathrm{C}$ und für die- 
jenigen aus der Nordsee auf $16^{\circ}$ bis $18^{\circ} \mathrm{C}$. Oft ist aber zwischen benachbarten Temperaturstufen eine gemeinsame Kompromißtemperatur durchaus möglich, z. B. $19^{\circ} \mathrm{C}$ für Mittelmeer- und Nordseetiere. Da mit steigender Temperatur der $\mathrm{O}_{2}$-Gehalt des Wassers sinkt, ist es zweckmäßig, mit der Temperatur eher im jeweiligen unteren Bereich zu bleiben und dabei eine langsamere Entwicklung in Kauf zu nehmen. Es wäre denkbar, daß manche Tierarten bei Simulation täglicher und vielleicht auch jahreszeitlicher Temperaturschwankungen besser gedeihen als bei konstanter Temperatur; jedoch ist darüber meines Wissens bisher nichts bekannt.

\section{Licht}

Auch die Beleuchtung spielt für das Gedeihen einer Zucht unter Umständen eine bedeutsame Rolle. In bezug auf die Lichtintensität können die Ansprüche zwar verschieden sein, doch tolerieren viele Tiere in dieser Hinsicht einen recht weiten Bereich. Unabhängig davon empfiehlt es sich allgemein, Kulturen, die lebende autotrophe Organismen als Futter enthalten, im Hinblick auf deren Photosynthese nicht zu dunkel aufzustellen; durch die Wahl einer geeigneten Lichtintensität gelingt es in manchen Fällen, für einige Zeit ein Gleichgewicht zwischen Vermehrung und Dezimierung des Futterorganismus einzustellen. Umgekehrt sollte man z. B. Hydroidenkulturen, die mit Artemia gefüttert werden und die dabei zwangsläufig einer ständigen Zufuhr unerwünschter autotropher Einzeller ausgesetzt sind, so dunkel wie möglich stellen, um die Entwicklung der störenden Organismen hintanzuhalten. Über einen Einfluß der Lichtqualität auf Zuchten von Meerestieren ist meines Wissens bisher nichts bekanntgeworden. Dagegen hat mit Sicherheit die Photoperiode in vielen Fällen große Bedeutung. Beispielsweise ist es bei der Zucht des Polychaeten Platynereis dumerilii unbedingt notwendig, die Fortpflanzungstermine der einzelnen Individuen zu synchronisieren, indem - sozusagen als Nachahmung des natürlichen Mondlichtzyklus die normalerweise auf täglich 12 bis 16 Stunden beschränkte Belichtung in jeder 4. Woche auch auf die Nachtzeit ausgedehnt wird (vgl. p. 260). Es braucht kaum ausdriicklich erwähnt zu werden, daß direkte Sonnenbestrahlung einer Kultur, allein schon wegen der damit verbundenen Erwärmung, immer schadet und daß deshalb zur Beleuchtung nur diffuses Tageslicht oder künstliches Licht von genügend weit entfernten Glühlampen oder Leuchtstoffröhren geeignet ist.

\section{Erhaltung eines biologischen Fließgleichgewichts}

Wegen der notwendigen Beschränkung der Zuchtbehälter auf eine Größenordnung, die im Vergleich zu den Wasservolumina im natürlichen Lebensraum verschwindend klein ist, kann sich in der Kultur - im Gegensatz zur freien Natur - unmöglich ein stabiles biologisches Gleichgewicht einstellen, bei dem die zu züchtenden Tiere ohne Eingriffe von außen beständig gedeihen. Solches wird in einer gut funktionierenden Zucht allein schon durch die alsbald auftretende Übervölkerung verhindert. Außerden erleidet das Seewasser, je nach seiner Belastung früher oder später, durch die Anhäu- 
fung von Endprodukten des tierischen Stoffwechsels Veränderungen, die in dem winzigen Volumen eines Zuchtgefäßes auf natürliche Weise, d. h. durch bakteriellen Abbau bzw. Verwertung dieser Produkte durch autotrophe Organismen nicht oder jedenfalls nicht schnell genug kompensiert werden können. Es bedarf deshalb in regelmäßigen Abständen künstlicher Eingriffe in eine Zucht, um die für ihr Gedeihen notwendigen Bedingungen innerhalb der Toleranzgrenzen zu halten: Man muß das Seewasser wechseln, eine Ansammlung von Exkrementen und Futterresten entfernen, fehlendes Futter ergänzen und den Vermehrungsüberschuß des gezüchteten Tieres abschöpfen. Die Zeitabstände, in denen diese konservierenden Maßnahmen durchzuführen sind, richten sich vor allem nach der Höhe des Futterumsatzes, nach der Populationsdichte und nach der Vermehrungsrate des Zuchttieres, und zwar sind sie diesen 3 Parametern umgekehrt proportional. Im allgemeinen liegen die Versorgungsintervalle im Bereich zwischen wenigen Tagen und einigen Wochen. In manchen Fällen lassen sich durch gewisse Kunstgriffe die Versorgungsintervalle verlängern. Bei größeren Kulturgefäßen, wie z. B. den erwähnten Polystyrolboxen, kann man verhältnismäßig leicht statt des periodischen Wasserwechsels eine kontinuierliche Durchströmung mit frischem Seewasser mittels einer für kleine Fördermengen geeigneten Pumpe vornehmen; allerdings kommt man dabei nicht umhin, den Wasserauslauf durch Gaze zu vergittern und dieselbe im Hinblick auf die Verstopfungsgefahr regelmäßig zu reinigen. Manche Hydroiden, wie z. B. Clytbia, wachsen nur gut, wenn sie in sehr kurzen Intervallen oder ständig Futter zur Verfügung haben. $\mathrm{Da}$ im Überschuß in die Kulturschale gegebene Artemia-Larven in kürzester Zeit durch die Cniden der Polypen getötet, in diesem Zustand später aber nicht mehr gefressen werden, kann man sich mit folgender Methode behelfen (Abb. 1/i). Man gibt in eine eigene Schale eine \pm große Anzahl von Artemia-Larven und verbindet dieses Vorratsgefäß über ein dünnes Heberglasrohr mit der Kulturschale, welche die Hydroiden enthält; beide Schalen stehen offen in einem geschlossenen Kühlschrankgefäß, und die ganze Anordnung wird auf der Schïttelapparatur bewegt. Bei richtiger Einstellung aller beteiligten Größen, wie Form und Volumen der Schalen, Wasserstand, Länge und Durchmesser des Glasrohrs, Frequenz und Richtung der Schüttelbewegung sowie Populationsdichte der Hydroiden und der Nauplien, gelangen laufend so viele Artemia-Larven durch das Glasrohr in die Hydroidenkultur, wie dort gefressen werden. Auf diese Weise ist über einen Zeitraum von 2 bis 3 Tagen eine automatische, kontinuierliche Futterversorgung möglich.

\section{Ein- und mehrgliedrige Kulturen}

Bisher wurde stillschweigend davon ausgegangen, daß die zu züchtende Tierart allein oder nur mit einem bestimmten Futterorganismus zusammen in der Kultur lebt, wenn man von den hier außer Betracht gelassenen Bakterien absieht. Tatsächlich sind in manchen Fällen solche Monokulturen durchaus möglich. Oftmals lassen sich aber in ciner Kultur stärker fördernde Bedingungen und stabilere Verhältnisse schaffen, wenn man in ihr mehrere Organismenarten vereinigt, die sich in ihren Ansprüchen und ihren Einwirkungen auf das Kulturgefüge gegenseitig ergänzen. So ist z. B. in dem System Platynereis/Platymonas die Anwesenheit einiger weiterer Tierarten, wie Oxyrrbis, gewisser Rotatorien und bestimmter Arten von hypotrichen Ciliaten für das Gedeihen 
der Polychaeten ausgesprochen förderlich, ja für gewisse frühe Stadien sogar unbedingt notwendig. Diese Begleitfauna hat in dem vorliegenden Fall eine doppelte Funktion: Einerseits verzehrt sie die anfallenden Bakterien, andererseits dient sie selbst den Polychaeten als Zusatznahrung. In Kulturen sessiler Tiere kann der Archiannelide Dinophilus nützlich sein, indem er die auf den Tierstöcken sich absetzenden Abfallstoffe frißt. Natürlich muß in jedem Einzelfall ausprobiert werden, welche Organismen in einer Kulturbiozönose auf die Dauer miteinander harmonieren. Man kann nicht hoffen, $\mathrm{daß}$ sich - etwa bei Verwendung nicht sterilisierten Seewassers - von selbst eine solche Gemeinschaft herausbildet; vielmehr würden auf solche Weise fast immer Organismen eingeschleppt, welche die Zucht durch Massenvermehrung früher oder später zugrunde richten.

\section{BEISPIELE WISSENSCHAFTLICHER UNTERSUCHUNGEN AN ZÜCHTBAREN MARINEN EVERTEBRATEN}

Im folgenden sollen einige, für verschiedene Forschungsrichtungen repräsentative Beispiele zeigen, welche Probleme der wissenschaftlichen Zoologie bisher an gezüchteten wirbellosen Meerestieren erfolgreich bearbeitet wurden. Eine Vollständigkeit der einschlägigen Literaturzitate ist hierbei nicht angestrebt.

Zahlreiche Untersuchungen sind z. B. an Hydrozoenkulturen durchgeführt worden. Ich selbst habe mich u. a. mit Hydractinia echinata beschäftigt und von dieser Art viele Klonkulturen jahrelang im Labor gezüchtet. Dabei ergaben sich Unterschiede in der Fähigkeit der einzelnen Klone, mit ihren Stolonen zu verschmelzen und auf diese Weise chimaerische Stöcke zu bilden. $\mathrm{Da}$ in den Laborzuchten auch geschlechtliche Nachzucht zu erhalten war, konnte ich in Kreuzungsversuchen die Erbfaktoren analysieren, welche die zwischen 2 Klonen bestehende Verträglichkeit oder Unverträglichkeit des Gewebes bedingen (HAuENSCHILD 1954, 1956a).

W. Müller $(1961,1964,1967,1969 a, b)$ hat am gleichen Objekt umfangreiche entwicklungsphysiologische Experimente zur Frage der Polypen- und Stockdifferenzierung unternommen; einige seiner Ergebnisse sind folgende: Die Planulalarven metamorphosieren nur in Kontakt mit bestimmten, das Substrat überziehenden Bakterienarten. Die Stacheln des Hydractinia-Stockes sind Wucherungen der Stolonenplatte, die durch äußere medhanische Reizung hervorgerufen werden. Aus zahlreichen Transplantations- und Regenerationsversuchen geht hervor, daß der abweichende Bau der Blastostyle durch ein am oralen Pol lokalisiertes Zentrum mit Induktorcharakter bestimmt wird und daß allgemein, auch bei den Hydranthen, für die Aufrechterhaltung der in einem Fließgleichgewicht befindlichen polaren Struktur ein morphogenetisches Gradientensystem bestimmter Art angenommen werden muß; biochemische Untersuchungen ergaben bereits erste Hinweise auf die stoffliche Natur dieses Systems. Die I-Zellen eines Stockes lassen sich durch Anwendung alkylierender Cytostatika selektiv ausschalten und mittels Transplantation eines Gewebestückes durch klonfremde I-Zellen ersetzen. Die Gonophore des Empfängerstockes bringen danach stets die Keimzellen hervor, die dem Geschlecht des I-Zellenspenders entsprechen. Bei Konkurrenz männlicher und weiblicher I-Zellen in einem chimaerischen Stock setzt sich infolge der höheren Vermehrungsrate der männlichen Gonien stets das männliche Erscheinungsbild vollständig 
und dauerhaft durch. WEILER-STOLT (1960) beschäftigte sich mit der Bedeutung der I-Zellen für die Fortpflanzung von Eleutheria und Cladonema. Sie fand bei ihren an Kulturen durchgeführten Untersuchungen, daß die Medusenknospen aus massiven I-Zellenansammlungen hervorgehen. In asexuellen Klonen, die im Verlauf der langjährigen vegetativen Zucht von Eleutheria-Medusen aufgetreten waren, konnte die Potenz zur Gametenbildung durch Ubertragung I-Zellen-reicher Gewebestückchen von normalen Spendern wiederhergestellt werden.

Werner $(1954,1956)$ hat bei den Hydroiden Margelopsis haeckeli und Ratbkea octopunctata und GüNzL (1964) bei Eirene viridula in Kulturversuchen festgestellt, daß für den Wechsel zwischen 2 verschiedenen Fortpflanzungsarten die Temperatur eine wichtige auslösende Rolle spielt. Die Margelopsis-Meduse bildet bei niedriger Temperatur hauptsächlich Subitaneier, bei höherer Temperatur dagegen überwiegend Dauereier. Bei der weiblichen Meduse von Ratbkea entstehen aus undifferenzierten Zellen des Manubriums bei niedriger Temperatur Medusenknospen, bei höherer Temperatur dagegen Eier. Bei Eirene induzierte die Überführung der gezüchteten Polypenstöcke aus $20^{\circ}$ in $29^{\circ} \mathrm{C}$ die Bildung zahlreicher Medusen und damit den Übergang von der vegetativen zur geschlechtlichen Fortpflanzung. Bei dem Hydroiden Eucheilota maculata war bis vor kurzem nur die Medusengeneration bekannt; WERNER (1968) konnte jetzt die Polypen aus befruchteten Eiern, die er von gefangenen Medusen erhielt, heranziehen und weiterzüchten und auf diese Weise die Artdiagnose vervollständigen. Dieses Beispiel zeigt, daß die Kulturmethode auch in den Dienst der Systematik gestellt werden kann, ein Gebiet, auf dem gerade bei den Hydrozoen noch zahlreiche offene Fragen einer Bearbeitung harren.

EMSCHERMANN (1961) züchtet seit vielen Jahren verschiedene Arten von Kamptozoen im Laboratorium. Bei Barentsia gracilis entdeckte er auf diese Weise das Vorkommen von gekammerten Brutkörpern; diese bilden sich aus keulig aufgetriebenen Stolonenspitzen, enthalten Reservestoffe und fungieren als Überdauerungsstadien.

An Laborzuchten der Nemertine Lineus sanguineus hat ReutTer (1967) Untersuchungen zur vegetativen Fortpflanzung durch Querteilung und zum Regenerationsvermögen durchgeführt. Es zeigten sich, z. B. bei der Regeneration in Extrakten verschiedener Körperbereiche, regionale Verschiedenheiten, die auf die mögliche Existenz eines stofflichen Gradienten hinweisen.

Bereits recht zahlreich sind die experimentellen Arbeiten, die an Polychaetenkulturen ausgeführt wurden. Sie betreffen, soweit dafür die Zucht im Labor mehr oder weniger als "conditio sine qua non" anzusehen ist, genetische Probleme, Fragen der Geschlechtsdifferenzierung, der lunaren Periodizität und der endokrinen Steuerung sowie die Phaenomene der Regeneration und des physiologischen Farbwechsels.

FøYN \& GJøEN (1954) konnten in Kreuzungsexperimenten die Vererbung des Farbmusters der Tentakelkrone von Pomatoceros triqueter auf ein einziges Allelpaar zurückführen; das Allel für Blau erwies sich als dominant, dasjenige für Braun als rezessiv. In ähnlicher Weise analysierte FISCHER (1969) eine in meinen Zuchten von Platynereis dumerilii spontan aufgetretene Augenfarb-Mutante; der Erbgang ist hier insofern von besonderem Interesse, als er mit Prädetermination verbunden ist. TRAUT (1968) entdeckte in seinen Kulturen von Dinophilus gyrociliatus zwei Mutanten, die gegenüber der Norm vergrößerte bzw. verkleinerte Männchen-Eier produzieren; auch 
hier wurde in beiden Fällen eine monofaktorielle Vererbung ermittelt, wobei für die Eigröße nicht der Genotyp des Eies selbst, sondern derjenige des Muttertieres maßgebend ist.

Im Anschluß an die älteren Kulturversuche von Hartmann et al. und von BaccI (Literatur bei H. MüLLER 1962) hat H. MüLLER (1962) die Frage der Geschlechtsdifferenzierung bei Opbryotrocha puerilis auf der Basis von Laborzuchten erneut aufgegriffen. Dabei trat einerseits die von HARTMANN in bezug auf das einzelne Individuum gefundene Modifizierbarkeit des sexuellen Differenzierungszustandes durch Außenfaktoren erneut zutage; in Paarkulturen zweier Weibchen trat die Rückverwandlung des einen Partners in ein Männchen allerdings nur ein, wenn sich beide Tiere berühren konnten, d. h. eine stoffliche Fernwirkung war nicht nachzuweisen. Andererseits bestätigte sich auf der Ebene der Population die von BACCI aufgezeigte Existenz genetischer Unterschiede in bezug auf die Reaktionsnorm der sexuellen Entwicklung; zwar wurden niemals reine Männchen oder Weibchen gefunden, jedoch konnten Stämme mit früher oder später einsetzender spontaner Geschlechtsumwandlung vom Männchen zum Weibchen herausgezüchtet werden. Ich selbst habe mich mit dem Problem der Geschlechtsbestimmung bei der Syllide Brania (=Grubea) clavata befaßt (HAUENSCHILD 1953, 1959). In diesem Fall differenziert sich von vornherein der eine Teil der Tiere weiblich, der andere männlich; das Geschlechtsverhältnis weicht meist stark von $1: 1 \mathrm{ab}$ und ist in den einzelnen Zuchten sehr verschieden. Während der männliche Zustand zeitlebens stabil bleibt, wandeln sich alle Weibchen früher oder später irreversibel in Sekundär-Männchen um; der Zeitpunkt dieses Geschlechtsumschlages wird maßgebend von Außenbedingungen mitbestimmt. In mehrjährigen Zuchtversuchen zeigte sich, daß bei fortgesetzter Kreuzung von Weibchen mit Sekundär-Männchen der Weibchen-Anteil in den Nachkommenschaften im Durchschnitt signifikant größer war als in einem Vergleichsstamm, in dem die Eier immer von Primär-Männchen besamt wurden; danach ist eine genetische Beeinflussung der Geschlechtsbestimmung auf polyfaktorieller Basis anzunehmen.

An dem Polychaeten Platynereis dumerilii, der heute bereits in 3 binnenländischen Instituten als Haustier gehalten wird, konnte ich erstmals die Ursachen einer lunaren Periodizität experimentell analysieren (Haunsschicd 1955, 1956b, 1961). Es stellte sich bei Versuchen mit künstlicher Beleuchtung im Laboratorium heraus, daß eine zyklische Veränderung der nächtlichen Belichtungsdauer, wie sie in freier Natur durch den Phasenwechsel des Mondes gegeben ist, Zeitgeberfunktion hat und die circalunaren endogenen Rhythmen der Einzelindividuen synchronisiert; dabei ist noch eine nächtliche Beleuchtungsstärke von weniger als 0,1 Lux wirksam. Die lunare Periodizität des Einzelindividuums zeigte sich zunächst nur an ihrer letzten Auswirkung und als ein kollektives Phänomen, indem zu bestimmten Zeiten des 30tägigen Mondoder Kunstlichtzyklus gehäuft reife Heteronereis schwärmten und sich fortpflanzten. Einige Jahre später erzielte NeumanN $(1966,1968)$ entsprechende Ergebnisse bei der Mücke Clunio marinus in bezug auf Schlïpfen und Fortpflanzung der Imagines. In seinen an Laborzuchten durchgeführten Untersuchungen gelang es ihm darüber hinaus, die lunare bzw. semilunare Schlüpfrhythmik von Clunio statt durch Licht auch durch einen künstlichen Gezeitenzyklus zu steuern; ferner ergaben sich bestimmte Beziehungen zwischen der Tagesrhythmik des Schlüpfens und dem Licht-Dunkel-Wechsel. 
Während bei Clunio vorerst noch nichts darüber bekannt ist, auf welchem Wege das Licht die Entwicklung im Sinne eines Zeitgebers beeinflußt, konnte für Platynereis eine Einschaltung des Hormonsystems in diesen Vorgang nachgewiesen werden ( $\mathrm{HAU}_{\mathrm{AU}}$ ENSCHILD 1956c, 1966). Ein im Prostomium wahrscheinlich von neurosekretorischen Zellen des Gehirns gebildetes Hormon hemmt beim juvenilen Wurm die geschlechtliche Entwicklung; erst wenn die Produktion dieses Hormons nachläßt und schließlich ganz aufhört, kommen Keimzellentwicklung und epitoke Metamorphose in Gang, und die Tiere schwärmen nach Ablauf dieser Prozesse als reife Heteronereis. Das Licht synchronisiert also bei allen in einem entsprechenden Entwicklungsstadium befindlichen Individuen primär den Verlauf der endokrinen Aktivität.

Konnten die bisher genannten Untersuchungen eigentlich nur auf der Basis von Laborzuchten überhaupt sinnvoll durchgeführt werden, so gibt es natürlich auch Fragestellungen, die prinzipiell durchaus an kurzfristig gehältertem Tiermaterial bearbeitet werden können. Auch in solchen Fällen bietet aber die Existenz von Kulturen oft entscheidende Vorteile und schafft verbesserte methodische Voraussetzungen. Besonders wichtig ist dabei die genetische Homogenität des Untersuchungsmaterials, wie sie bei Klonkulturen und bis zu einem gewissen Grade auch bei fortgesetzter geschlechtlicher Inzucht gegeben ist; bedeutsam ist außerdem, daß die Vorgeschichte jedes einzelnen Versuchstieres genau bekannt ist und daß im gezüchteten Material keine saisonbedingten Verschiedenheiten auftreten.

Bei der caudalen Regeneration von Platynereis konnte auf Grund dieser Vorteile nicht nur gezeigt werden, daß dieser Vorgang an das Vorhandensein des Prostomiums gebunden ist, sondern es ließ sich mit Hilfe von Kopftransplantationen und UV-Strahlenstichoperationen erstmalig auch beweisen, daß der Einfluß des Prostomiums auf die Regeneration hormonaler Natur ist (Hauenschild 1960, Hofmann 1966). Fischer (1965) entdeckte an gezüchtetem Material eine durch den Licht-Dunkel-Wechsel gesteuerte Tagesrhythmik des physiologischen Farbwechsels bei Platynereis dumerilii und wies eine endogene Komponente derselben nach. RösELER $(1967,1970)$ gelang es dann, auch eine endogene lunare Periodizität des Farbwechsels festzustellen und außerdem den Nachweis zu erbringen, daß die wechselnde Ausbreitung des Pigments in den Chromatophoren sowohl nervös durch cholinerge Nerven als auch hormonal reguliert wird.

Zum Schluß sollen 2 Beispiele zeigen, daß Laborkulturen von wirbellosen Meerestieren auch günstige Voraussetzungen für stoffwechsel- und sinnesphysiologische Arbeiten bieten können. Provasoli et al. (1959) beschäftigten sich unter ernährungsphysiologischen Gesichtspunkten mit der monoxenischen Kultur des Copepoden Tigriopus; sie konnten dabei Daten über den essentiellen Nährstoffbedarf erarbeiten und die Auswirkungen einer einseitigen Ernährung mit nur einem einzigen Futterorganismus über mehrere Generationen hinweg studieren. SCHNEIDER $(1955,1959)$ untersuchte an Kulturen des Bryozoons Bugula avicularia, die er mit einer von Grell entwickelten Methode jahrelang im Labor hielt, die Prinzipien des Stockaufbaues und den Phototropismus der Knospen, für den er bestimmte Zellelemente in der Knospenkuppe verantwortlich machen konnte.

Sicher kann die Methode, Meerestiere nicht nur in Meeresstationen sondern auch in binnenländischen Laboratorien fortlaufend zu züchten, in Zukunft noch viele weitere experimentelle Untersuchungen hervorbringen. Sie wird vermutlich auch mithelfen, 
eine auf manchen Gebieten durch Festlegung auf wenige Standardobjekte drohende Erstarrung zu überwinden; denn nur eine Vielzahl von Objekten aus den verschiedensten systematischen Kategorien wird, vom Problem und von der Methode her, der Vielgestaltigkeit der Lebenserscheinungen und ihrer Erforschung gerecht.

\section{ZUSAMMENFASSUNG}

1. Im Rahmen eines Übersichtsreferates werden einige allgemeine Prinzipien der Laboratoriumszucht mariner Wirbelloser abgehandelt.

2. Als Voraussetzungen einer kontinuierlichen Zucht werden u. a. herausgestellt: die Auswahl geeigneter Arten und Zuchtgefäße, die Berïcksichtigung der für das Gedeihen entscheidenden Umweltfaktoren und Maßnahmen zur Aufrechterhaltung des biologischen Gleichgewichtes in einer Kultur.

3. Die Bedeutung der Zucht wirbelloser Meerestiere für die experimentelle Zoologie wird anhand einiger Beispiele wissenschaftlicher Untersuchungen illustriert.

\section{ZITIERTE LITERATUR}

Emschermann, P., 1961. Uber Brutkörper bei dem Kamptozoon Barentsia gracilis. Zool. Jb. (Abt. allg. Zool. Physiol. Tiere) 69, 333-338.

Frscher, A., 1965. Uber die Chromatophoren und den Farbwechsel bei dem Polychaeten Platynereis dumerilii. Z. Zellforsch. mikrosk. Anat. 65, 290-312.

- 1969. Eine Pigmentmangelmutante bei dem Polychaeten Platynereis dumerilii. Mol. gen. Genet. 104, 360-370.

FøYN, B. \& GJøen, I., 1954. Studies on the Serpulid Pomatoceros triqueter: II. The colour pattern of the branchial crown and its inheritance. Nytt Mag. Zool. 2, 85-90.

GüNZL, H., 1964. Untersuchungen über die Auslösung der Medusenknospung bei Hydroidpolypen. Zool. Jb. (Abt. Anat. Ontogenie Tiere) 81, 491-528.

HAUENSCHILD, C., 1953. Die phaenotypische Geschlechtsbestimmung bei Grubea clavata und vergleichende Beobachtungen an anderen Sylliden. Zool. Jb. (Abt. allg. Zool. Physiol. Tiere) 64, 14-54.

- 1954. Genetische und entwicklungsphysiologische Untersuchungen über Intersexualität und Gewebeverträglichkeit bei Hydractinia echinata. Wilhelm Roux Arch. EntzeMech. Org. 147, 1-41.

- 1955. Photoperiodizität als Ursache des von der Mondphase abhängigen MetamorphoseRhythmus bei dem Polychaeten Platynereis dumerilii. Z. Naturf. (B) 10, 658-662.

- 1956a. Über die Vererbung einer Gewebeverträglichkeits-Eigenschaft bei dem Hydroidpolypen Hydractinia echinata. Z. Naturf. (B) 11, 132-138.

- 1956b. Neue experimentelle Untersuchungen zum Problem der Lunarperiodizität. Naturwissenschaften 43, 361-363.

- 1956c. Hormonale Hemmung der Geschlechtsreife und Metamorphose bei dem Polychaeten Platynereis dumerilii. Z. Naturf. (B) 11, 125-132.

- 1959. Weitere Kreuzungsversuche zur Frage der Geschlechtsbestimmung bei dem Polychaeten Grubea clavata. Z. Naturf. (B) 14, 89-92.

- 1960. Abhängigkeit der Regenerationsleistung von der inneren Sekretion im Prostomium bei Platynereis dumerilii. Z. Naturf. (B) 15, 52-55.

- 1961. Die Schwärmperiodizität von Platynereis dumerilii im DD/LD-Belichtungszyklus und nach Augenausschaltung. Z. Naturf. (B) 16, 753-756.

- 1962. Die Zucht mariner Wirbelloser im Laboratorium (Methoden und Anwendung). Kieler Meeresforsch. 18 (Sonderheft), 28-37. 
- 1966. Der hormonale Einfluß des Gehirns auf die sexuelle Entwicklung bei dem Polychaeten Platynereis dumerilii. J. Endocr. 6, 26-73.

- 1968. Hälterung und Laboratoriumszuchten von Invertebraten. In: Methoden der meeresbiologischen Forschung. Hrsg. von C. Schlieper VEB G. Fischer, Jena, 192-209.

HofmanN, D. K., 1966. Untersuchungen zur Regeneration des Hinterendes bei Platynereis dumerilii. Zool. Jb. (Abt. allg. Zool. Physiol, Tiere) 72, 374-430.

MÜlLER, H., 1962. Über die Sexualität des Polychaeten Ophryotrocha puerilis, ihre Determination und ihren Einfluß auf Drüsentätigkeit und Kauapparatentwicklung. Z. Morph. Ökol. Tiere 52, 1-32.

MÜLLER, W., 1961. Untersuchungen zur Stockdifferenzierung von Hydractinia echinata. Zool. Jb. (Abt. allg. Zool. Physiol. Tiere) 69, 317-324.

- 1964. Experimentelle Untersuchungen über Stockentwicklung, Polypendifferenzierung und Sexualchimaeren bei Hydractinia ecbinata. Wilbelm Roux Arch. EntwMech. Org. 155, 181-268.

- 1967. Differenzierungspotenzen und Geschlechtsstabilität der I-Zellen von Hydractinia ecbinata. Wilhelm Roux Arch. EntreMech. Org. 159, 412-432.

- 1969a. Auslösung der Metamorphose durch Bakterien bei den Larven von Hydractinia ecbinata. Zool. Jb. (Abt. Anat. Ontogenie Tiere) 86, 84-95.

- 1969b. Die Steuerung des morphogenetischen Fließgleichgewichts in den Polypen von $\mathrm{Hy}$ dractinia echinata. I. Biologisch-experimentelle Untersuchungen. Wilhelm Roux Arch. EntwMech. Org. 163, 334-356.

Neumann, D., 1966. Die lunare und tägliche Schlüpfperiodik der Mücke Clunio (Steuerung und Abstimmung auf die Gezeitenperiodik). Z. vergl. Physiol. 53, 1-61.

- 1968. Die Steuerung einer semilunaren Schlüpfperiodik mit Hilfe eines künstlichen Gezeitenzyklus. Z. vergl. Physiol. 60, 63-78.

Provasoli, L., Shiraishi, K. \& Lance, J. R., 1959. Nutritional idiosyncrasies of Artemia and Tigriopus in monoxenic culture. Ann. N. Y. Acad. Sci. 77, 250-261.

ReUtTER, K., 1967. Untersuchungen zur ungeschlechtlichen Fortpflanzung und zum Regenerationsvermögen von Lineus sanguineus. Wilhelm Roux Arch. EntwMech. Org. 159, 141-202.

Röseler, I., 1967. Versuche über die Periodizität der Fortpflanzung und des Chromatophorenspiels bei dem Polychaeten Platynereis dumerilii. Unveröff. Dipl.-Arb., Freiburg/Br.

- 1970. Untersuchungen über den physiologischen Farbwechsel bei dem Polychaeten Platynereis dumerilii. Zool. Anz. (Suppl. Bd) 33 (im Druck).

Schneider, D., 1955. Phototropisches Wachstum der Zoide und Rhizoide von Bugula avicularia. Naturwissenschaften 42, 48-49.

- 1959. Der Aufbau der Bugula-Tierstödke und seine Beeinflussung durch Außenfaktoren. Biol. Zbl. 78, 250-283.

Traut, W., 1968. Genetische Analyse zweier Mutanten von Dinophilus gyrociliatus mit veränderter Eigröße. Helgoländer wiss. Meeresunters. 18, 296-316.

WeILER-Stolt, B., 1960. Uber die Bedeutung der interstitiellen Zellen für die Entwidklung und Fortpflanzung mariner Hydroiden. Wilhelm Roux Arch. EntwMech. Org. 152, 398-455.

WERNER, B., 1954. Über die Fortpflanzung der Anthomeduse Margelopsis baeckeli durch Subitan- und Dauereier und die Abhängigkeit ihrer Bildung von äußeren Faktoren. Zool. Anz. (Suppl. Bd) 18, 124-133.

- 1956. Über die entwicklungsphysiologische Bedeutung des Fortpflanzungswechsels der Anthomeduse Rathkea octopunctata. Zool. Anz. 156, 159-177.

- 1968. Polypengeneration und Entwicklungsgeschichte von Eucheilota maculata. (Mit einem Beitrag zur Methodik der Kultur mariner Hydroiden.) Helgoländer wiss. Meeresunters. 18, 136-168.

Anschrift des Autors: Prof. Dr. C. Hauenschild

Zoologisches Institut

der Technischen Universität

33 Braunschweig

Pockelsstr. 10a

Deutschland (BRD) 